\title{
Multilevel Comprehensive Evaluation and Decision Making of Ergonomics
}

\author{
Wen-jun Wang, Sui-huai Yu, Shu-xia Wang, Junxuan Chen, and Li Deng \\ Key Laboratory of Contemporary Design and Integrated Manufacturing Technology, Northwestern Polytechnical University, \\ Xian 710072, China \\ Correspondence should be addressed to Shu-xia Wang; 2008wangshuxia@163.com
}

Received 25 September 2014; Revised 15 March 2015; Accepted 16 March 2015

Academic Editor: Peng Shi

Copyright (C) 2015 Wen-jun Wang et al. This is an open access article distributed under the Creative Commons Attribution License, which permits unrestricted use, distribution, and reproduction in any medium, provided the original work is properly cited.

Due to the improvement of living standards, higher level of ergonomic performance is required for the products. As a result, the ergonomic evaluation has become one of the key points in the decisions making of modern products, especially the complex products. Aiming at improving the situation that the methods for ergonomic evaluation and decision making are one-sided and discrete, this paper proposed several methods for measuring multiply factors and data format of products and built a comprehensive evaluation and decision making system. In this system, the data supplied by soft hardware and specialists were calculated separately to gain the preliminary scores, and the preliminary scores were processed to get the final results for the decision making using the AHP-GRA analytic hierarchy process-gray relational analysis method proposed in this paper.

\section{Introduction}

The products always change with the development of the society. At an early year of design, designers pay almost all of their attention into the realization of the functions and the function or capability of a product is the most important element in deciding whether the product is good or not. In recent years, the factor of ergonomic has drawn more and more attention of the users, and the products are required to be more humanized to satisfy the customers.

As a result, the designers have to do a lot of work on ergonomic from all angles, and the component of ergonomic can be complicated. It is always difficult for the decision makers to evaluate several proposals to find out the best one in the development process, because there are too many factors to be taken into consideration. Lots of companies and institutions have been developing software and hardware to simulate the using situation or to record data in the reality, such as the Motion Analysis, Tekscan, and Applied Science Laboratories. This equipment supplies plenty of information of the human body when using the product and can reflect the ergonomic performance in some way. On the other hand, the designers and the decision makers often have a good sense of the ergonomic performance of the product and they always judge the proposals with their experiences. The former solution for ergonomic evaluation cannot simulate perfectly and the data of a single piece of equipment can only reflect certain factors, while the latter one contains subjective elements and it is not accurate enough.

This paper proposed an evaluation system that combines the subjective and the objective evaluation to supply a comprehensive solution for decision making on ergonomics. Based on the entropy weight method and improved TOPSIS method, the data of a single piece of equipment can be processed to get the close-degree of each proposal to the positive ideal solution. The fuzzy evaluation method can be applied to process the data supplied by specialists, and the score for each proposal can be acquired. The close-degree and the score stand for the ergonomic levels and they can be put together to figure out the final decision applying a new method.

The remainder of this paper is organized as follows. In Section 2, the related works on ergonomic evaluation is discussed. Our method for ergonomic evaluation and decision making is presented in Section 3, in which we firstly build a tree of ergonomic evaluation and present the evaluation processing of soft hardware data and the fuzzy evaluation processing of the specialists' subjective score, and finally 
combine the results gained above to make the comprehensive evaluation and get the final result. Section 4 reports the implementation of the method and shows examples about ergonomic evaluation of cockpit. The conclusion is drawn in Section 5.

\section{Related Works}

Plenty of research about ergonomic evaluation has been done by scholars from all over the world. In general, the existing research focused on three points, and the first one is research on how to evaluate specific items by using software or hardware, which paid most attention to the processing of data. The second type of research focuses on building system for the ergonomic evaluation and the structure of the system is the key point. The last type of research is about how to evaluate the ergonomics according to the score supplied by specialists, and the processing method always plays the most important part.

On the first type of research, the instruments are used to supply data. Ramsis was used to provide score labeled the Ramsis discomfort value to develop an optimal TGS lever envelop based on human characteristics [1]. Eye tracking system was used to investigate whether human eye movement dynamics can predict computer-based assessment performance in different presentation modalities. The study added empirical evidence of a prediction model between eye movement behaviors and successful cognitive performance [2]. The EMG (electromyography) instrument was applied to calculate signals under different accelerations in the simulated dynamic driving environment to evaluate human comfort under different dynamic driving environment [3]. On the second type of research, the Delphi is the most used method to build the evaluation index. Research on using the Delphi method to build the evaluation indexes for unmanned aerial vehicles ground control station, which can meet the demand and can be used as the guidance for ergonomic evaluation [4], and building evaluation index system based on modified Delphi method to evaluate the helicopter display interface [5] have been carried out. When it comes to the third type of research on ergonomic evaluation, the specialists play an important part and the MADM (multiple attribute decision making) and the fuzzy evaluation are the most used methods in the current research. Systems and theories of ergonomic evaluation based on fuzzy MADM have been developed [6-8].

Although the current research about ergonomic evaluation is one-sided and uncomprehensive, they cover a lot of fields and can supply basic method for this paper.

\section{A New Method for Ergonomic Evaluation and Decision Making}

3.1. Tree of Ergonomic Evaluation. Generally, the data used to evaluate the ergonomics can be divided into two kinds, the accurate data and the fuzzy data. The accurate data is acquired through software that can simulate the using situation of the products such as Jack, Ramsis, Delmia, and hardware that can measure certain physiological index of human body, such as the Eye-Track System, the Pressure Scan System, the EMG, and the BioNex. The fuzzy data is always supplied by specialists that have good understanding of the product.

Due to the huge difference between the two kinds of data, separate processing method has to be used. This paper built an ergonomic evaluation tree, shown in Figure 1, to state the evaluation system. For the soft hardware, this paper will improve the existing method to get the result of each software or hardware, and for the specialists, the fuzzy data will be calculated using fuzzy evaluation method. Finally, the scores will be joined together to gain the ranking of all the proposals and decisions that can be made.

3.2. Evaluation of Soft Hardware Based on Entropy Weight Method and Improved TOPSIS Method. The soft hardware can supply huge number of data, and they must be classified into different indexes. Here in this paper, the number of the index was assumed to be $n$, and the proposal number was assumed to be $m$. All the data of different index and proposal formed the evaluation matrix $X$. Consider

$$
X=\begin{gathered}
Q_{1} \\
Q_{2} \\
\vdots \\
Q_{m}
\end{gathered}\left[\begin{array}{cccc}
X_{11} & X_{12} & \cdots & X_{1 n} \\
X_{21} & X_{22} & \cdots & X_{2 n} \\
\vdots & \vdots & X_{i j} & \vdots \\
X_{m 1} & X_{m 2} & \cdots & X_{m n}
\end{array}\right] .
$$

The process was carried out as in the following steps.

Step 1 (the standardization of the matrix). The data can be divided into two kinds, the cost-type and the benefit-type. The cost-type means the greater the number, the worse the proposal, and the benefit-type is opposite. The evaluation matrix must be standardized before the next calculation by the method as follows.

For the benefit-type,

$$
a_{i j}=\frac{X_{i j}}{\sqrt{\sum_{i=1}^{n} X_{i j}^{2}}} .
$$

For the cost-type,

$$
a_{i j}=\frac{1}{X_{i j} \sqrt{\sum_{i=1}^{n}\left(1 / X_{i j}\right)^{2}}} .
$$

The standard matrix $A$ was acquired. Consider

$$
A=\begin{gathered}
Q_{1} \\
Q_{2} \\
\vdots \\
Q_{m}
\end{gathered}\left[\begin{array}{cccc}
a_{11} & a_{12} & \cdots & a_{1 n} \\
a_{21} & a_{22} & \cdots & a_{2 n} \\
\vdots & \vdots & a_{i j} & \vdots \\
a_{n 1} & a_{n 2} & \cdots & a_{n n}
\end{array}\right]
$$

Step 2. Assigning weights to each index using entropy weight method $[9,10]$. According to the definition of entropy in 


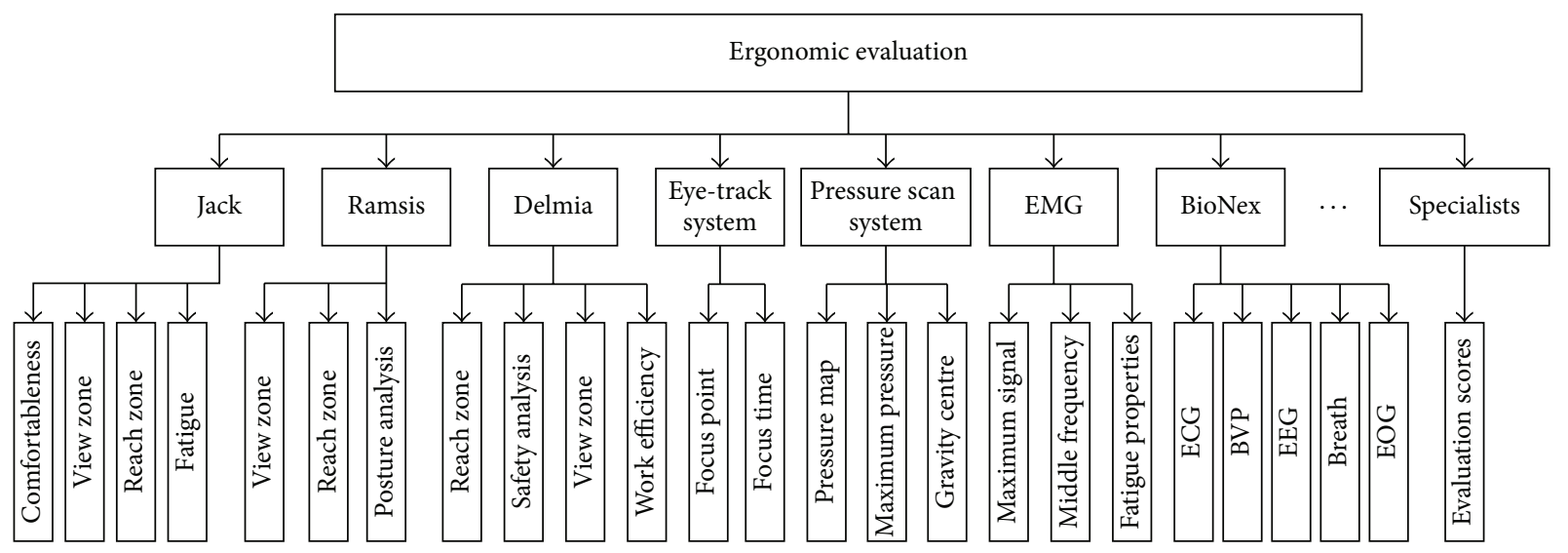

FIgURE 1: Tree of ergonomic evaluation.

chemistry, the entropy of the index $j$ in $A$ was defined as follows:

$$
H_{j}=-\frac{\sum_{i=1}^{m} a_{i j} \ln a_{i j}}{\ln m} .
$$

The weight of the index $j$ was $w_{j}$

$$
w_{j}=\frac{1-H_{j}}{n-\sum_{i=1}^{n} H_{j}} .
$$

Step 3. Constructing the decision-making matrix $Z$

$$
\begin{aligned}
Z & =\left(z_{i j}\right)_{m \times n}=\left(w_{j} \cdot a_{i j}\right)_{m \times n} \\
& =\left[\begin{array}{cccc}
w_{1} \cdot a_{11} & w_{2} \cdot a_{12} & \ldots & w_{n} \cdot a_{1 n} \\
w_{1} \cdot a_{21} & w_{2} \cdot a_{22} & \ldots & w_{n} \cdot a_{2 n} \\
\vdots & \vdots & w_{j} \cdot a_{i j} & \vdots \\
w_{1} \cdot a_{m 1} & w_{2} \cdot a_{m 2} & \ldots & w_{n} \cdot a_{m n}
\end{array}\right] .
\end{aligned}
$$

Step 4. Calculating the positive ideal solution and the negative ideal solution

$$
\begin{aligned}
& z^{+}=\max _{i=1}^{m} z_{i j}=\left(z_{i 1}{ }^{+}, z_{i 2}{ }^{+}, \ldots, z_{i n}{ }^{+}\right), \\
& z^{-}=\min _{i=1}^{m} z_{i j}=\left(z_{i 1}{ }^{-}, z_{i 2}{ }^{-}, \ldots, z_{i n}{ }^{-}\right) .
\end{aligned}
$$

Step 5. Calculating the distance of each proposal to the positive ideal solution and the negative ideal solution, because the data in $Z$ is smaller than 1, the square in the Euclidean distance will make it smaller, and the distance will not be exact. This paper used the Canberra distance instead of the Euclidean distance to calculate the distance. The Canberra distance was proposed by Lance and Williams, and it was widely used in cluster analysis. The definition of Canberra distance is as follows, and it is clear that it is better dealing with small number:

$$
d_{i j}(L)=\frac{1}{n} \sum_{t=1}^{n} \frac{\left|x_{i t}-x_{j t}\right|}{x_{i t}+x_{j t}} \quad(i, j=1,2, \ldots, m) .
$$

Then the distance to the positive ideal solution and the negative ideal solution can be gained

$$
S_{i}^{+}=\frac{1}{n} \sum_{j=1}^{n} \frac{\left|x_{i j}-x_{j}^{+}\right|}{x_{i j}+x_{j}^{+}}, \quad S_{i}^{-}=\frac{1}{n} \sum_{j=1}^{n} \frac{\left|x_{i j}-x_{j}^{-}\right|}{x_{i j}+x_{j}^{-}} .
$$

Step 6. Calculating the close-degree of each proposal to the positive ideal solution $A_{i}$ [8]:

$$
A_{i}=\frac{S_{i}^{-}}{S_{i}^{+}+S_{i}^{-}} \text {. }
$$

Step 7. Obtaining the results, $A^{\prime}=\left(A_{1}^{\prime}, A_{2}^{\prime}, \ldots, A_{m}^{\prime}\right)$.

The same process can be applied to all the other soft hardware.

3.3. Fuzzy Evaluation of Specialists' Subjective Score. A lot of research has been done on evaluation about the specialists that participate in the decision making, of which the MADM was the most attractive one. Plentiful mathematical methods were proposed to support the MADM as well, such as the AWA (addition weighted average), OWA (order weighted average), and TOPSIS. The MADM can be divided into accurate MADM and fuzzy MADM according to the data type processed. Due to the complexity and uncertainty of the ergonomics, people often cannot offer accurate score even the specialists. As a result, this paper will apply the fuzzy MADM to proceed the evaluation.

To express the subjective score of the specialists, the interval number must be defined. If $\widetilde{s}=\left[s^{a}, s^{b}\right]=\left\{x \mid s^{a} \leq\right.$ $\left.x \leq s^{b}\right\}$, it can be defined as an interval number, and $s^{b}, s^{a}$ will be the upper limit and the lower limit, respectively. Several definitions of the interval number are as follows.

The exponentiation of the interval number:

$$
\left[s^{a}, s^{b}\right]^{\lambda}=\left[\left(s^{a}\right)^{\lambda},\left(s^{b}\right)^{\lambda}\right] .
$$

The multiplication of the interval number:

$$
\widetilde{s_{1}} \cdot \widetilde{s_{2}}=\left[s_{1}^{a}, s_{1}^{b}\right] \cdot\left[s_{2}^{a}, s_{2}^{b}\right]=\left[s_{1}^{a} \cdot s_{2}^{a}, s_{1}^{b} \cdot s_{2}^{b}\right] .
$$


TABLE 1: Transform from linguistic variables into IFN.

\begin{tabular}{lc}
\hline Linguistic variables & IFN \\
\hline Very important & $(0.90,0.05,0.05)$ \\
Important & $(0.75,0.20,0.05)$ \\
Medium & $(0.50,0.40,0.10)$ \\
Unimportant & $(0.25,0.60,0.15)$ \\
Very unimportant & $(0.10,0.80,0.10)$ \\
\hline
\end{tabular}

The length of the interval number:

$$
l(\widetilde{s})=s^{b}-s^{a} .
$$

The possibility degree of the interval number [11]:

$$
p=\frac{\max \left\{0, l_{1}+l_{2}-\max \left(s_{2}^{b}-s_{1}^{a}, 0\right)\right\}}{l_{1}+l_{2}} .
$$

3.3.1. Assigning Weight for the Specialists. The specialists are of different majors, levels, and experiences, which will affect their understanding of the proposal. Thus, different weight must be assigned to different specialists. Article [12] of the references offered a method to calculate the weight of each specialist in a group based on subjective weight assignment, which can solve the problem of weight assignment in this paper.

This method was based on the definition of the IFS (Intuitionistic Fuzzy Set).

If $A=\left\{\left\langle x, \mu_{A}(x), v_{A}(x)\right\rangle\right\} \in X$ and $X$ is nonnull, $\mu_{A}(x)$ standing for the membership degree of the element $x$ in $X$ belonging to $A, v_{A}(x)$ standing for the nonmembership degree, and $0 \leq \mu_{A}(x)+v_{A}(x) \leq 1, A$ will be defined as an IFS on $X$.

Based on the definition of IFS, the IFN (Intuitionistic Fuzzy Set) can be defined. If $\pi_{A}=1-\mu_{A}-v_{A}$, the array $\alpha=\left(\mu_{A}, v_{A}, \pi_{A}\right)$ can be named IFN $[12,13]$.

The linguistic variables used to describe the specialists can be transferred into IFNs as Table 1 (The scores of IFN were an example, and they can change slightly according to the situation).

Assuming the number of specialists is $t$, the weight of each one of them can be calculated using the equation as follows [14]:

$$
\lambda_{k}=\frac{\left[\mu_{k}+\pi_{k}\left(\mu_{k} /\left(\mu_{k}+v_{k}\right)\right)\right]}{\sum_{i=1}^{t}\left[\mu_{k}+\pi_{k}\left(\mu_{k} /\left(\mu_{k}+v_{k}\right)\right)\right]} \quad(k=1,2, \ldots, t) .
$$

It can be known that $\sum_{k=1}^{t} \lambda_{k}=1$, which means that this method assigned the whole weight in the form of a percentage.

3.3.2. Concentration of the Evaluation Data. The concentration means to combine the data and the weight to simplify the matrix.
The scores offered by the specialists were put together to form the decision-making matrix. After the standardization, the standard matrix $R_{k}$ was gained:

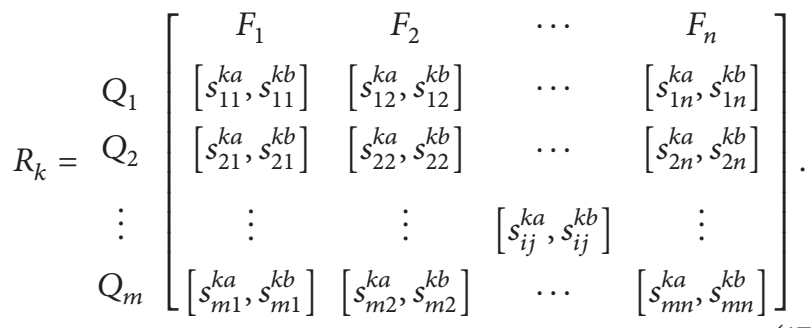

Calculated using (3), the weights of specialists were computed with the scores to get the 2 nd generation decisionmaking matrix $R_{m}[15]$ :

$$
\begin{aligned}
R_{m} & =\left(r_{i j}\right)_{m \times n}=\left(\prod_{i=1}^{t}\left[s_{i j}^{k a}, s_{i j}^{k b}\right]^{\lambda_{k}}\right)_{m \times n} \\
& =\left(\prod_{i=1}^{t}\left(s_{i j}^{k a}\right)^{\lambda_{k}}, \prod_{i=1}^{t}\left(s_{i j}^{k b}\right)^{\lambda_{k}}\right)_{m \times n} .
\end{aligned}
$$

Assuming that the weight vector of the factors of the products calculated using AHP was $W=\left(\alpha_{1}, \alpha_{2}, \ldots, \alpha_{n}\right)$, the second concentration was proceeded to get the final decisionmaking matrix $R_{f}$ as the first step:

$$
\begin{aligned}
R_{f} & =\left(f_{i}\right)_{m \times 1} \\
& =\prod_{\tau=1}^{n}\left(\left[\prod_{i=1}^{t}\left(s_{i j}^{k a}\right)^{\lambda_{k}}, \prod_{i=1}^{t}\left(s_{i j}^{k b}\right)^{\lambda_{k}}\right]^{\alpha_{\tau}}\right)_{m \times 1} .
\end{aligned}
$$

After that, the complementary judgment matrix $P$ was built by the data in $R_{f}$ using (15):

$$
P=\left[\begin{array}{ccccc}
0.5 & p_{12} & p_{13} & \cdots & p_{1 m} \\
1-p_{12} & 0.5 & p_{23} & \cdots & p_{2 m} \\
1-p_{13} & 1-p_{23} & 0.5 & \cdots & p_{3 m} \\
\vdots & \vdots & \vdots & 0.5 & \vdots \\
1-p_{1 m} & 1-p_{2 m} & 1-p_{3 m} & \cdots & 0.5
\end{array}\right]
$$

Adding all the data of each line in $P$, the final result of the evaluation of specialists $B^{\prime}=\left(B_{1}^{\prime}, B_{2}^{\prime}, \ldots, B_{m}^{\prime}\right)$ was acquired, in which $B_{i}^{\prime}=\sum_{j=1}^{m}\left(P_{i j}\right)$.

3.4. Comprehensive Evaluation and Decision Making. To get the final result of the evaluation, the results in Sections 3.2 and 
3.3 were combined to build the new decision-making matrix F

$$
F=\left(F_{i j}\right)_{m \times(l+1)}=Q_{2}\left[\begin{array}{ccccc}
G_{1} & G_{2} & \cdots & G_{l} & G_{l+1} \\
A_{11} & A_{12} & \cdots & A_{1 l} & B_{1} \\
A_{21} & A_{22} & \cdots & A_{2 l} & B_{2} \\
\vdots & \vdots & A_{i j} & \vdots & \vdots \\
A_{m 1} & A_{m 2} & \cdots & A_{m l} & B_{m}
\end{array}\right] .
$$

Assigning weight for the software and specialists is complicated because the weight changes in different situations. This paper divided the process into two parts, the subjective part and the objective part, and proposed a new method AHP-GRA to solve the problem.

Assuming that the importance $G_{i}$ relative to $G_{j}$ was $J_{i j}=$ $G_{i} / G_{j}$, the judge matrix $J$ was built

$$
J=\left[\begin{array}{ccccc}
1 & J_{12} & J_{13} & \cdots & J_{1(l+1)} \\
\frac{1}{J_{12}} & 1 & J_{23} & \cdots & J_{2(l+1)} \\
\frac{1}{J_{13}} & \frac{1}{J_{23}} & 1 & \cdots & \vdots \\
\vdots & \vdots & \vdots & 1 & J_{1 l} \\
\frac{1}{J_{1(l+1)}} & \frac{1}{J_{2(l+1)}} & \cdots & \frac{1}{J_{1 l}} & 1
\end{array}\right] .
$$

After checking the consistency of $J$, the subjective weight of each factor in $F$ was acquired [16]

$$
\overline{w_{i}^{\prime}}=\sqrt[(l+1)]{\prod_{j=1}^{l+1} J_{i j}} \quad(i, j=1,2, \ldots, l+1) .
$$

Standardizing the weight to get $w^{\prime}=\left(w_{1}^{\prime}, w_{2}^{\prime}, \ldots, w_{l+1}^{\prime}\right)$ by the computing as follows:

$$
w_{i}^{\prime}=\frac{\overline{w_{i}^{\prime}}}{\sum_{j=1}^{l+1} \overline{w_{j}^{\prime}}} \quad(i=1,2, \ldots, l+1) .
$$

Finding the largest score $\rho$ in $F$ and building the referenced vector $\theta=(\rho, \rho, \ldots, \rho)$ and then calculating the distance between the data in $F$ and $\theta$ [17]:

$$
D_{j}=\sum_{i=1}^{m}\left(\rho-F_{i j}\right)^{2} \quad(j=1,2, \ldots, l+1) .
$$

Amending the distance with $w^{\prime}$

$$
D_{j}^{\prime}=\frac{D_{j}}{w_{j}^{\prime}} \quad(j=1,2, \ldots, l+1) .
$$

The weight of each factor in $F$ was acquired by $w_{j}=1 /(1+$ $\left.D_{j}^{\prime}\right)$. After the standardization through $w_{j}^{*}=w_{j} / \sum_{i=1}^{l+1} w_{i}$, the final weight $w^{*}=\left(w_{1}^{*}, w_{2}^{*}, \ldots, w_{l+1}^{*}\right)$ can be acquired.
The index $\mu(x)$ contains the decision-making data and the weight. The index before and after the amendment is as follows:

$$
\begin{aligned}
& \mu_{0}(j)=\sum_{i=1}^{n} F_{i j} w_{j}^{\prime}, \\
& \mu(j)=\sum_{i=1}^{n} F_{i j} w_{j}^{*} .
\end{aligned}
$$

Then calculating the distance between $\mu_{0}(j)$ and $\mu(j)$ :

$$
L\left(\mu, \mu_{0}\right)=\sqrt{\sum_{j=1}^{l+1}\left(\mu(j)-\mu_{0}(j)\right)^{2}} .
$$

If $L$ is smaller than the set threshold $\varepsilon$, the weight is good to use. Otherwise, the value of $w^{*}$ will be transferred to $w^{\prime}$, and the process above will be carried out again until $L<\varepsilon$.

It is clear that the final weight involves both the subjective and the objective elements and it can balance the factors better.

After getting the weight of each factor in $F$, the same calculated process in Section 3 can be applied to acquire the final results of each proposal, and the ranking of them will tell which one is the best in ergonomic.

\section{Implementation and Examples}

In a cooperative project with an aircraft design organization, four proposals of the cockpit were supplied to make the decision. To evaluate these proposals, this paper applied Jack software, the ASL Eye-track 6 System, and the Tekscan Pressure Scan System to do the evaluation separately. And 4 specialists including 3 pilots and a designer of aircraft were invited to evaluate these proposals.

4.1. Processing of Soft Hardware. The Jack software is developed by University of Pennsylvania and it supplies plenty of tools for ergonomic evaluation. After lightweight and transformation of the $3 \mathrm{D}$ models, the four proposals were imported into Jack. Due to the confidentiality of this project, the models have to be simplified as shown in Figure 2.

In this paper, the tool of view zone and reach zone analysis was used to calculate the number of components inside or outside the range of view zones and reach zones. At the same time, the method of RULA (rapid upper limb assessment) and the Lower Back Analysis were applied to gain the RULA score and the L4/L5 forces. The evaluation results of Jack are shown in Table 2.

The results were put together to build the evaluation matrix $X$ :

$$
X=\begin{aligned}
& Q_{1} \\
& Q_{2} \\
& Q_{3} \\
& Q_{4}
\end{aligned}\left[\begin{array}{lllll}
1 & 2 & 7 & 593 & 3 \\
1 & 3 & 8 & 420 & 4 \\
3 & 4 & 7 & 338 & 3 \\
2 & 2 & 7 & 411 & 5
\end{array}\right] .
$$




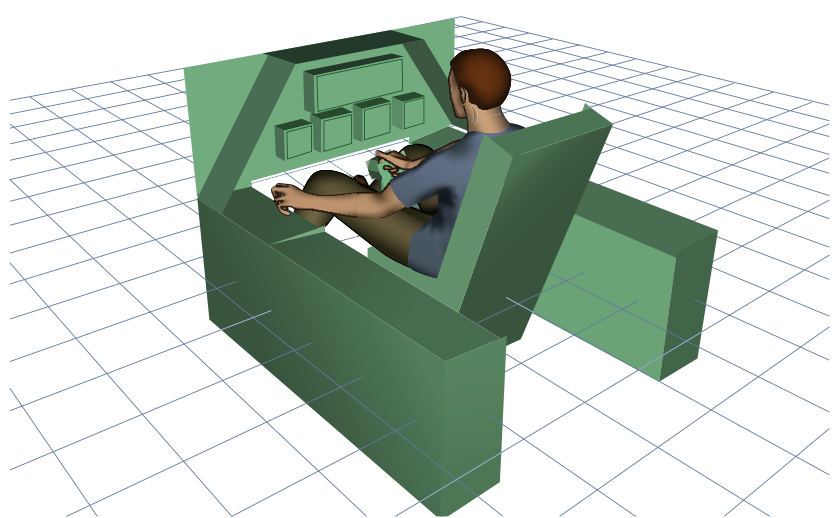

$Q_{1}$

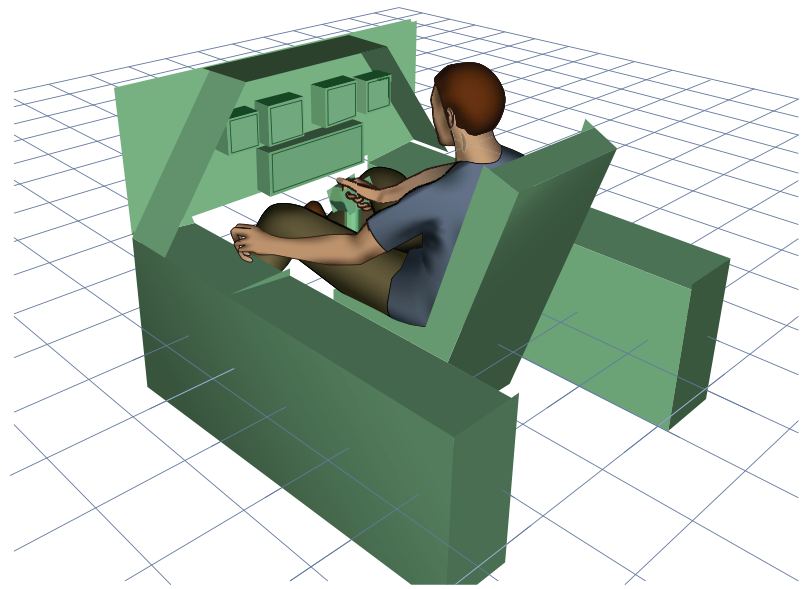

$Q_{3}$

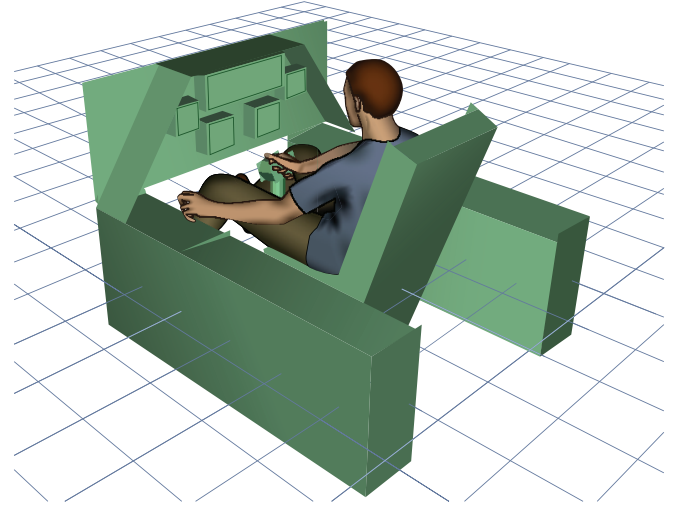

$Q_{2}$

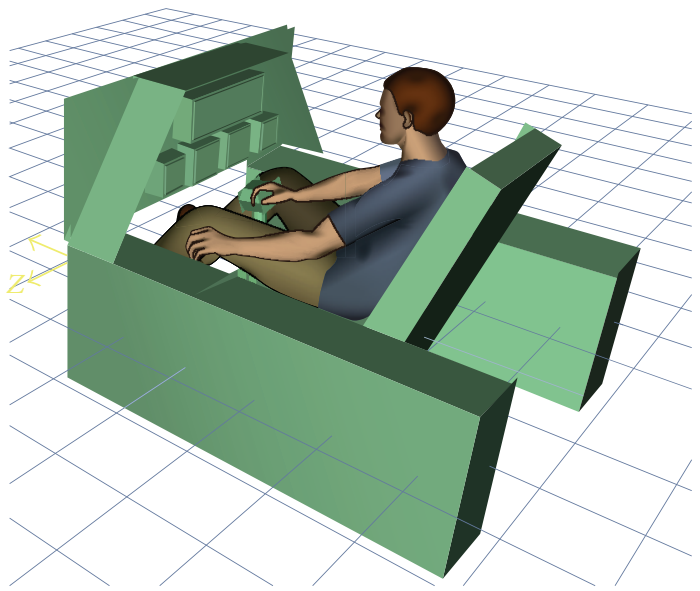

$Q_{4}$

FIGURE 2: Four proposals of aircraft cockpit in Jack.

TABLE 2: Evaluation results of Jack.

\begin{tabular}{lccccc}
\hline Proposals & $\begin{array}{c}\text { Number of controllers out } \\
\text { of the range of the hands' } \\
\text { reach zone }\end{array}$ & $\begin{array}{c}\text { Number of displayers out of } \\
\text { the range of the view zone }\end{array}$ & $\begin{array}{c}\text { Number of buttons in the } \\
\text { range of thumb's reach zone }\end{array}$ & L4/L5 Forces & Score of RULA \\
\hline 1 & 1 & 2 & 7 & 393 & 420 \\
2 & 1 & 3 & 7 & 438 & 3 \\
4 & 3 & 4 & 7 & 411 \\
\hline
\end{tabular}

Standardizing the matrix using (2) and (3) to get the standard matrix $A$ :

$$
A=\begin{aligned}
& Q_{1} \\
& Q_{2} \\
& Q_{3} \\
& Q_{4}
\end{aligned}\left[\begin{array}{lllll}
0.35 & 0.32 & 0.24 & 0.18 & 0.30 \\
0.35 & 0.21 & 0.28 & 0.25 & 0.23 \\
0.12 & 0.16 & 0.24 & 0.31 & 0.30 \\
0.18 & 0.32 & 0.24 & 0.25 & 0.18
\end{array}\right]
$$

The entropy of each index can be calculated by using (5):

$$
\begin{gathered}
H_{1}=0.936, \quad H_{2}=0.974, \quad H_{3}=0.998, \\
H_{4}=0.985, \quad H_{5}=0.988 .
\end{gathered}
$$

The weight of each index can be acquired using (6):

$$
\begin{gathered}
w_{1}=0.0726, \quad w_{2}=0.0295, \quad w_{3}=0.0023, \\
w_{4}=0.0170, \quad w_{5}=0.0136 .
\end{gathered}
$$

The decision-making matrix $Z$ :

$$
Z=\begin{aligned}
& Q_{1} \\
& Q_{2} \\
& Q_{3} \\
& Q_{4}
\end{aligned}\left[\begin{array}{lllll}
0.0254 & 0.0094 & 0.0006 & 0.0031 & 0.0041 \\
0.0254 & 0.0062 & 0.0007 & 0.0041 & 0.0031 \\
0.0087 & 0.0047 & 0.0006 & 0.0053 & 0.0041 \\
0.0131 & 0.0094 & 0.0006 & 0.0041 & 0.0024
\end{array}\right] .
$$


TABLE 3: Linguistic variables and IFN of the specialists.

\begin{tabular}{lcc}
\hline Specialists & Linguistic variables & IFN \\
\hline 1 & Medium & $(0.45,0.35,0.20)$ \\
2 & Important & $(0.75,0.20,0.05)$ \\
3 & Very important & $(0.90,0.05,0.05)$ \\
4 & Medium & $(0.55,0.35,0.10)$ \\
\hline
\end{tabular}

Finding the positive solution and the negative solution:

$$
\begin{aligned}
& z^{+}=(0.0254,0.0094,0.0007,0.0053,0.0041) \\
& z^{-}=(0.0087,0.0062,0.0006,0.0031,0.0024)
\end{aligned}
$$

Calculating the Canberra distance and then the closedegree using (10) and (11):

$$
A_{1}^{\prime}=(0.738,0.638,0.300,0.411)
$$

The same process can be applied to acquire the results of the ASL Eye-Track 6 System and the Tekscan System:

$$
\begin{aligned}
& A_{2}^{\prime}=(0.532,0.281,0.421,0.732), \\
& A_{3}^{\prime}=(0.522,0.634,0.347,0.139) .
\end{aligned}
$$

4.2. Fuzzy Evaluation of Specialists. The importance of the 4 specialists was decided by their experience and ages and the linguistic variables and IFN were listed in Table 3.

The weight of each specialist can be calculated using (16):

$$
\begin{aligned}
& \lambda_{1}=0.193, \lambda_{2}=0.271, \quad \lambda_{3}=0.326, \\
& \lambda_{4}=0.210 .
\end{aligned}
$$

In the evaluation of the cockpit, the upper and lower limits of the interval numbers were set from 1 to 7 , standing for the ergonomic levels from "very bad" to "very good." The scores of the ejection seat, the layout of the displayers, the layout and structure of the controllers, and the color of the cockpit offered by the specialist were put together to build $R_{1} \sim R_{4}$ :

$$
\begin{aligned}
& R_{1}=\begin{array}{l}
Q_{1} \\
Q_{2} \\
Q_{3} \\
Q_{4}
\end{array}\left[\begin{array}{llll}
{[5,6]} & {[4,6]} & {[5,7]} & {[5,6]} \\
{[6,7]} & {[3,6]} & {[3,5]} & {[5,7]} \\
{[4,6]} & {[5,6]} & {[6,7]} & {[4,5]}
\end{array}\right], \\
& R_{2}=\begin{array}{l}
Q_{1} \\
Q_{2} \\
Q_{3} \\
Q_{4}
\end{array}\left[\begin{array}{llll}
{[4,7]} & {[4,5]} & {[5,6]} & {[6,7]} \\
{[5,6]} & {[3,7]} & {[4,5]} & {[5,6]} \\
{[3,6]} & {[5,7]} & {[5,6]} & {[4,6]}
\end{array}\right],
\end{aligned}
$$

$$
\begin{aligned}
& R_{3}=\begin{array}{l}
Q_{1} \\
Q_{2} \\
Q_{3} \\
Q_{4}
\end{array}\left[\begin{array}{llll}
{[4,6]} & {[5,7]} & {[3,6]} & {[5,6]} \\
{[3,7]} & {[3,6]} & {[4,5]} & {[4,6]} \\
{[5,6]} & {[6,7]} & {[5,7]} & {[4,5]}
\end{array}\right], \\
& R_{4}=\begin{array}{l}
Q_{1} \\
Q_{2} \\
Q_{3} \\
Q_{4}
\end{array}\left[\begin{array}{llll}
{[3,5]} & {[5,6]} & {[4,6]} & {[5,7]} \\
{[4,5]} & {[4,6]} & {[3,5]} & {[6,7]} \\
{[3,6]} & {[4,7]} & {[5,6]} & {[3,6]}
\end{array}\right] .
\end{aligned}
$$

The 2nd generation decision-making matrix $R_{m}$ can be acquired using (18):

$$
\begin{aligned}
& R_{m} \\
& =\begin{array}{l}
Q_{1} \\
Q_{2} \\
Q_{3} \\
Q_{4}
\end{array}\left[\begin{array}{lllll}
{[3.93,5.97]} & {[4.53,6.03]} & {[4.04,6.22]} & {[5.24,6.40]} \\
{[3.19,6.30]} & {[3.20,6.23]} & {[3.58,4.99]} & {[4.83,6.39]} \\
{[3.77,6.01]} & {[5.01,6.76]} & {[5.17,6.57]} & {[3.78,5.46]}
\end{array}\right] .
\end{aligned}
$$

The weight of the ejection seat, the layout of the displayers, the layout and structure of the controllers, and the color of the cockpit were $W=(0.292,0.234,0.357,0.117)$, and the final decision-making matrix $R_{f}$ was acquired using (19):

$$
R_{f}=\left[\begin{array}{l}
{[4.24,6.12]} \\
{[4.22,5.96]} \\
{[3.78,5.79]} \\
{[4.52,6.30]}
\end{array}\right]
$$

Applying (15) to build the complementary judgment matrix $P$ :

$$
P=\left[\begin{array}{llll}
0.5000 & 0.525 & 0.601 & 0.437 \\
0.475 & 0.500 & 0.581 & 0.409 \\
0.399 & 0.419 & 0.500 & 0.335 \\
0.563 & 0.591 & 0.665 & 0.500
\end{array}\right]
$$

The final results of the specialists were acquired:

$$
B^{\prime}=(2.063,1.965,1.653,2.319) .
$$

4.3. Comprehensive Evaluation and Decision Making. Applying the results in Sections 4.1 and 4.2 to build the new 
decision-making matrix $F^{\prime}$ and standardize the matrix using (2) and (3) to get the standard matrix $F$ :

$$
\begin{aligned}
& F^{\prime}=\begin{array}{l}
Q_{1} \\
Q_{2} \\
Q_{3} \\
Q_{4}
\end{array}\left[\begin{array}{llll}
0.738 & 0.532 & 0.522 & 2.063 \\
0.638 & 0.281 & 0.634 & 1.965 \\
0.300 & 0.421 & 0.347 & 1.653 \\
0.411 & 0.732 & 0.139 & 2.319
\end{array}\right], \\
& F=\begin{array}{l}
Q_{1} \\
Q_{2} \\
Q_{3} \\
Q_{4}
\end{array}\left[\begin{array}{llll}
0.354 & 0.271 & 0.318 & 0.258 \\
0.306 & 0.143 & 0.386 & 0.245 \\
0.144 & 0.214 & 0.211 & 0.207 \\
0.197 & 0.372 & 0.085 & 0.290
\end{array}\right] .
\end{aligned}
$$

The judge matrix $J$ was built according to the importance:

$$
J=\left[\begin{array}{cccc}
1 & 5 & 7 & 2 \\
\frac{1}{5} & 1 & 2 & \frac{1}{2} \\
\frac{1}{7} & \frac{1}{2} & 1 & \frac{1}{3} \\
\frac{1}{2} & 2 & 3 & 1
\end{array}\right] .
$$

The consistency of $J$ is good and there is no need to correct it. The subjective weight of each factor can be calculated using (23):

$$
\begin{aligned}
& \overline{w_{1}}=\sqrt[4]{1 \times 5 \times 7 \times 2}=2.893, \\
& \overline{w_{2}}=\sqrt[4]{\frac{1}{5} \times 1 \times 2 \times \frac{1}{2}}=0.669, \\
& \overline{w_{3}}=\sqrt[4]{\frac{1}{7} \times \frac{1}{2} \times 1 \times \frac{1}{3}}=0.393, \\
& \overline{w_{4}}=\sqrt[4]{\frac{1}{2} \times 2 \times 3 \times 1}=1.316 .
\end{aligned}
$$

Standardizing the subjective weight to get $w^{\prime}$ :

$$
w^{\prime}=(0.549,0.127,0.075,0.250) \text {. }
$$

Setting the reference data $\theta=(0.386,0.386,0.386,0.386)$ and calculating the distance between the data in $F$ and $\theta$ :

$$
\begin{aligned}
D_{1}=0.102, \quad D_{2} & =0.102, \quad D_{3}=0.126, \\
D_{4} & =0.078 .
\end{aligned}
$$

Amending the distance

$$
\begin{aligned}
D_{1}^{\prime}=0.186, \quad & D_{2}^{\prime}=0.803, \quad D_{3}^{\prime}=1.68, \\
D_{4}^{\prime}=0.312 . &
\end{aligned}
$$

Then the final weight can be acquired:

$$
\begin{gathered}
w_{1}=0.843, \quad w_{2}=0.555, \quad w_{3}=0.373, \\
w_{4}=0.762
\end{gathered}
$$

Then the final weight of each factor can be acquired:

$$
\begin{aligned}
w_{1}^{*}=0.333, \quad w_{2}^{*} & =0.219, \quad w_{3}^{*}=0.147 \\
w_{4}^{*} & =0.301 .
\end{aligned}
$$

Set the threshold $\varepsilon=0.1$, and it can be calculated that $L=0.031<\varepsilon$. The final weight is good.

Building the decision-making matrix $Z_{f}$ with $F$ and the weight

$$
Z_{f}=\left[\begin{array}{llll}
0.118 & 0.059 & 0.047 & 0.078 \\
0.102 & 0.031 & 0.058 & 0.074 \\
0.048 & 0.047 & 0.031 & 0.062 \\
0.066 & 0.082 & 0.012 & 0.087
\end{array}\right]
$$

Finding the positive solution and the negative solution

$$
\begin{aligned}
& z_{f}{ }^{+}=(0.118,0.082,0.058,0.087), \\
& z_{f}{ }^{-}=(0.048,0.031,0.012,0.062) .
\end{aligned}
$$

Calculating the Canberra distance and then the closedegree using (10) and (11)

$$
\begin{gathered}
A_{\mathrm{Q} 1}=0.817, \quad A_{\mathrm{Q} 2}=0.646, \quad A_{\mathrm{Q} 3}=0.358, \\
A_{\mathrm{Q} 4}=0.453 .
\end{gathered}
$$

From the ranking of close-degree, it can be known that the best proposal on ergonomics is $Q_{1}$. The final result was approved by the aircraft design organization and the scores of each factors obtained during the process was coincident with their attitudes.

\section{Conclusions}

Involving subjective feeling, comfortableness, reaching zones and so on, the ergonomics evaluation of products is complicated, and lots of different methods can be applied. From the global perspective, this paper proposed a comprehensive method that can combine almost all the soft hardware that are related to ergonomics and the specialists to help dealing with the decision-making problem among certain proposals. Compared to traditional ones, this method is more detailed and of more operability, and it can improve and speed up the ergonomic evaluation and decision making in the product development.

\section{Conflict of Interests}

The authors declare that there is no conflict of interests regarding the publication of this paper.

\section{Acknowledgments}

This work is supported by the National 111 Project (Grant no. B13044) and the National Natural Science Foundation of China (51105310). 


\section{References}

[1] J. Nam, Y. Lee, and Y. Lim, "A study on optimal TGS lever envelopes based on human characteristics," SAE Technical Papers, 2013.

[2] S.-C. Chen, H.-C. She, M.-H. Chuang, J.-Y. Wu, J.-L. Tsai, and T.-P. Jung, "Eye movements predict students' computerbased assessment performance of physics concepts in different presentation modalities," Computers and Education, vol. 74, pp. 61-72, 2014.

[3] E. Zhang, C.-R. Bi, G.-H. Wang et al., "Experimental research on biological signal of muscle fatigue under dynamic driving environment," Journal of Engineering Design, vol. 17, no. 4, pp. 246-252, 2010.

[4] S.-J. Qi, T. Wang, and F. Wang, "Research on ergonomic integrated evaluations of UAV ground control station," Advances in Aeronautical Science and Engineering, vol. 4, no. 4, pp. 474-479, 2013.

[5] Z. Guo, D. Guo, J.-C. Yang, and C. Huang, "Study on evaluation index system of helicopter display interface ergonomics," Electronics Optics \& Control, vol. 18, no. 5, pp. 67-84, 2011.

[6] S.-B. Zhang, Research on Man-machine Interactive Evaluation System based on Multi-attribute Decision and Group Decision, Shandong Jianzhu University, 2012.

[7] Z. Lu and Y. Sun, "Maintainability evaluation model based on fuzzy multiple attribute decision making theory for virtual products," China Mechanical Engineering, vol. 20, no. 24, pp. 2978-2983, 2009.

[8] S. Wan and J. Dong, "A possibility degree method for intervalvalued intuitionistic fuzzy multi-attribute group decision making," Journal of Computer and System Sciences, vol. 80, no. 1, pp. 237-256, 2014.

[9] Y.-Y. Wang, H. Ren, H.-B. Wang et al., "Evaluation of energysaving building program based on entropy weight and TOPSIS method," Journal of Shandong Jianzhu University, vol. 18, no. 4, 2013.

[10] J.-Z. Bao, Study on Models of Multi-criteria Group Decisionmaking towards Formal Safety Assessment, Dalian Maritime University, 2011.

[11] Z.-S. Xu, Study on Methods for Multiple Attribute Decision Making under some Situations, Southeast University, Nanjing, China, 2002.

[12] J.-Q. Wang and Z. Zhang, "Multi-criteria decision-making method with incomplete certain information based on intuitionistic fuzzy number," Control and Decision, vol. 23, no. 10, pp. 1145-1148, 2008.

[13] S.-F. Zhang, Several Methods for Fuzzy Multiple Attribute Decision-Making with Applications, Xidian University, 2012.

[14] I. K. Vlachos and G. D. Sergiadis, "Intuitionistic fuzzy information-applications to pattern recognition," Pattern Recognition Letters, vol. 28, no. 2, pp. 197-206, 2007.

[15] G.-W. Wei, "Method of uncertain linguistic multiple attribute group decision making based on dependent aggregation operators," Systems Engineering and Electronics, vol. 32, no. 4, pp. 764769, 2010.

[16] L.-Y. Zhu, Research on the key technology of customer requirements processing of design for mass customization [Ph.D. thesis], Hefei University of Technology, 2008.

[17] J. Cui, Y.-G. Dang, and S.-F. Liu, "An improved approach for determining weights of attributes in decision making based on grey incidence," Chinese Journal of Management Science, vol. 16, no. 5, pp. 141-145, 2008. 


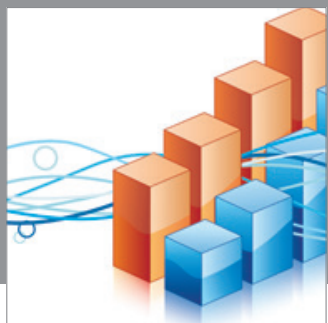

Advances in

Operations Research

mansans

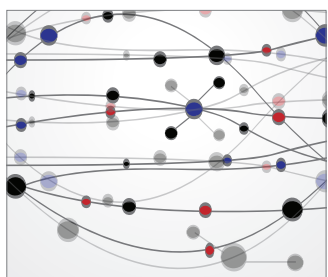

The Scientific World Journal
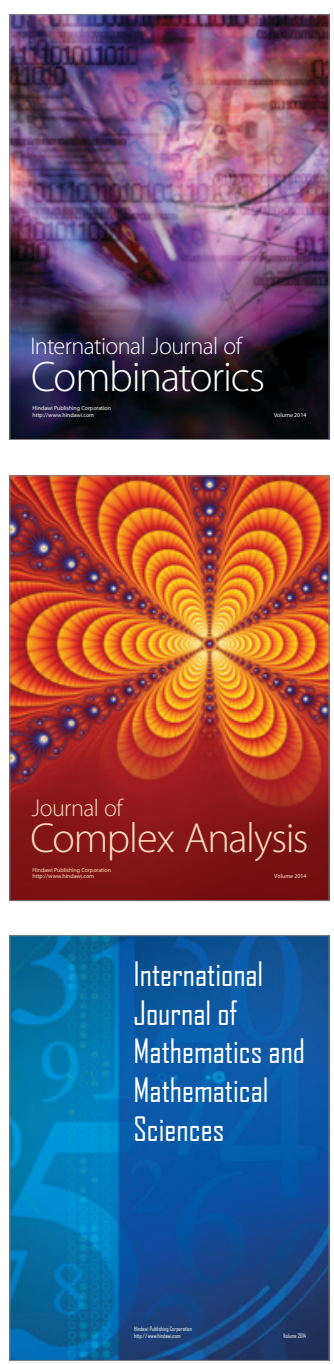
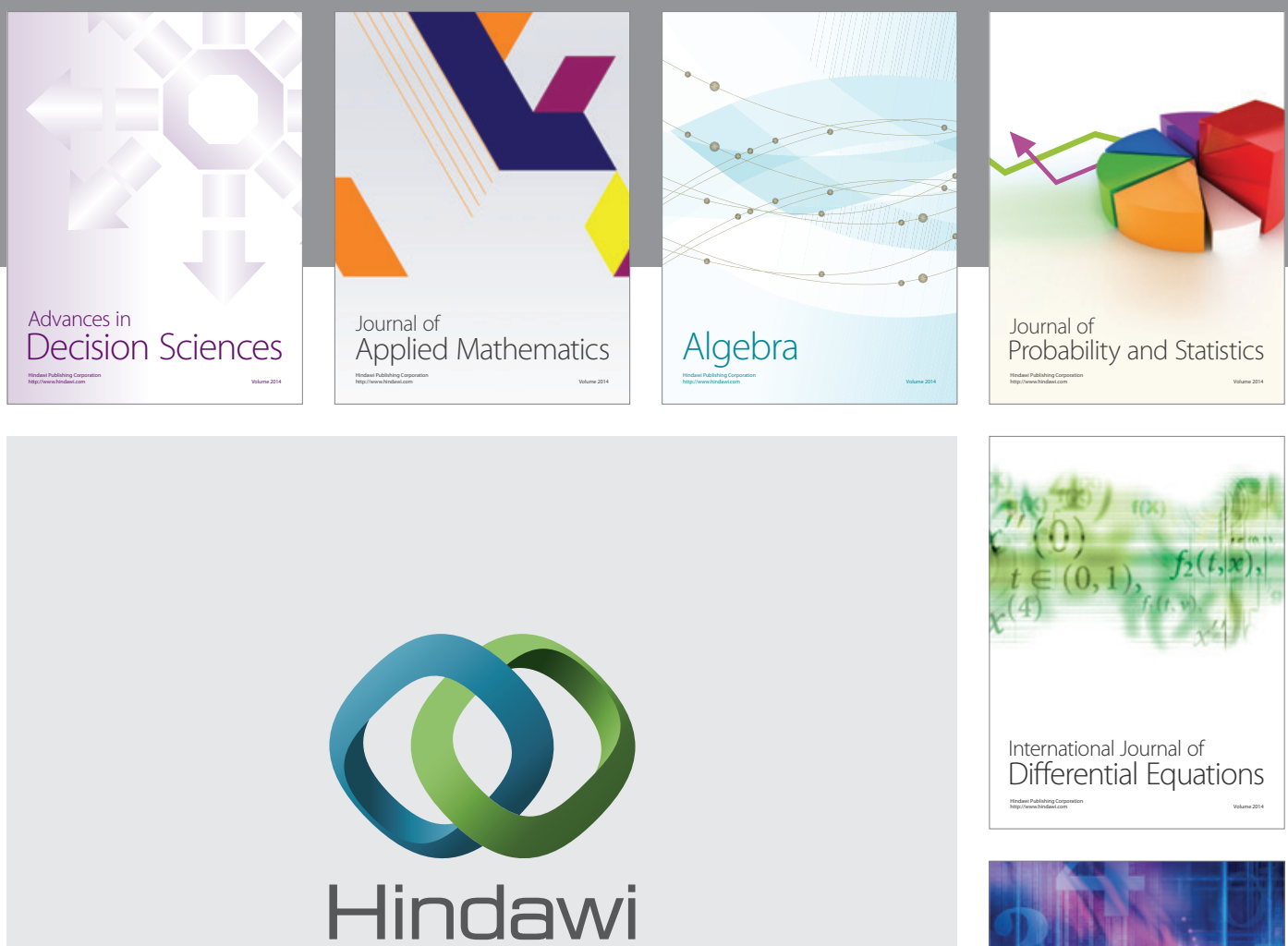

Submit your manuscripts at http://www.hindawi.com
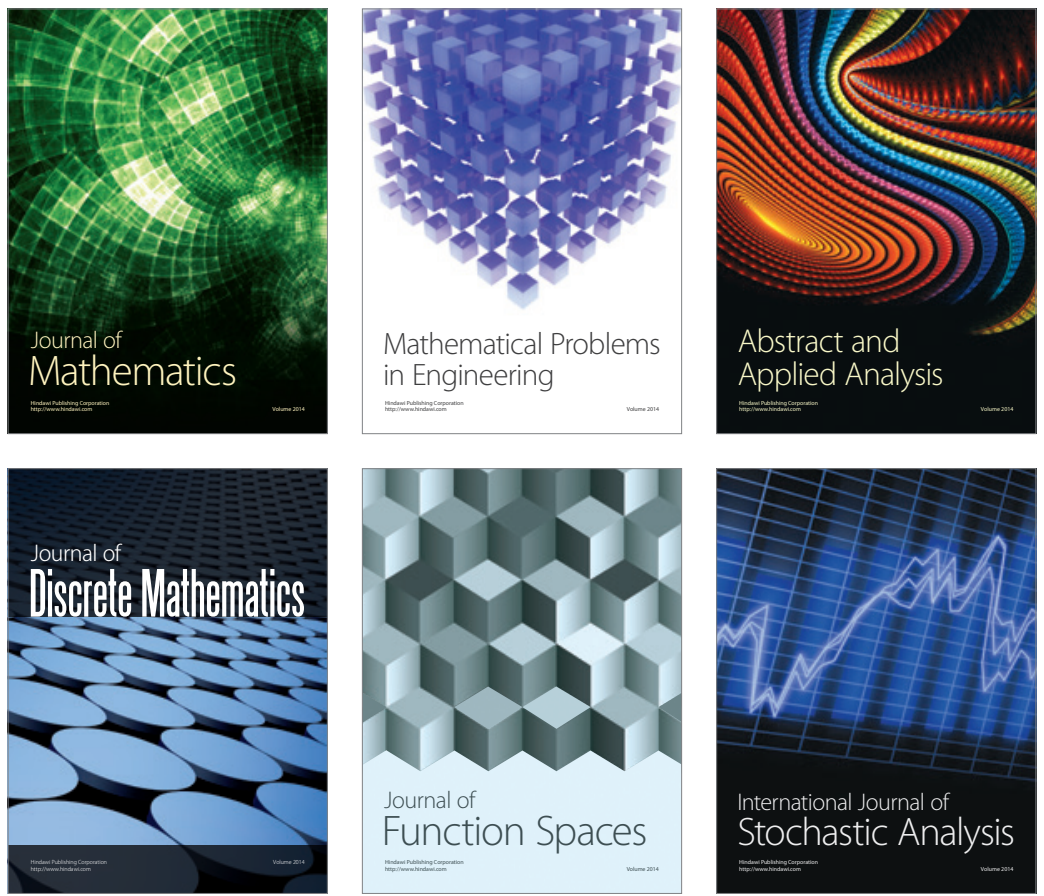

Journal of

Function Spaces

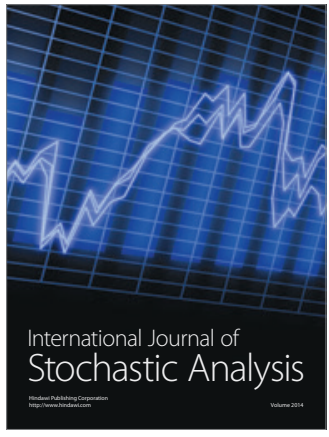

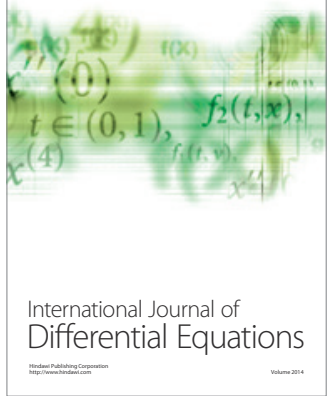
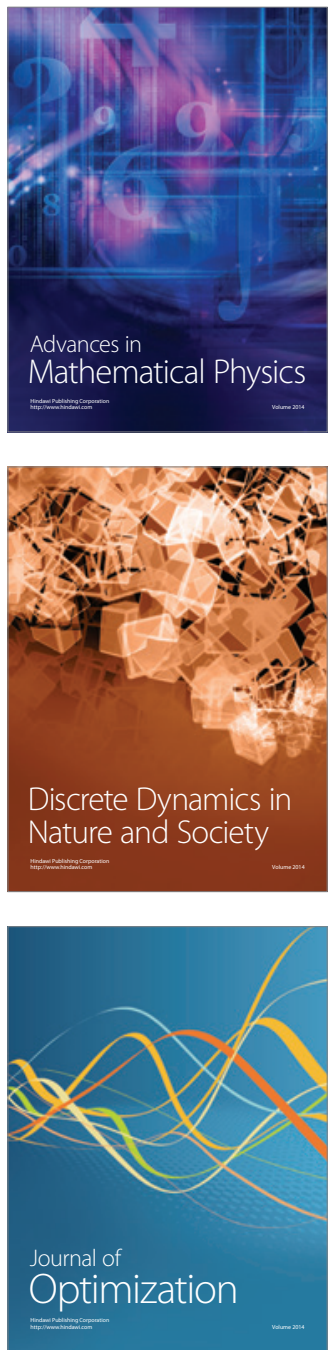